\title{
A randomized placebo-controlled trial of paroxetine for the management of anxiety in chronic obstructive pulmonary disease (PAC Study)
}

This article was published in the following Dove Press journal: Journal of Multidisciplinary Healthcare

\author{
Zafar A Usmani ${ }^{1,2}$ \\ Kristin V \\ Carson-Chahhoud ${ }^{1,3}$ \\ Adrian J Esterman ${ }^{4,5}$ \\ Brian J Smith ${ }^{1,2}$
}

'School of Medicine, The University of Adelaide, Adelaide, SA, Australia; ${ }^{2}$ Department of Respiratory Medicine, The Queen Elizabeth Hospital, Adelaide, SA, Australia; ${ }^{3}$ School of Health Sciences, The University of South Australia, Adelaide, SA, Australia; ${ }^{4}$ School of Nursing and Midwifery, The University of South Australia, Adelaide, SA, Australia; ${ }^{5}$ Australian Institute of Tropical Health Medicine, James Cook University, Cairns, QLD, Australia
Correspondence: Zafar A Usmani Department of Respiratory Medicine, The Queen Elizabeth Hospital, 4A, 28 Woodville Road, Woodville South, Adelaide, SA 50I I, Australia

Tel +6I 882226670

Fax +6I 88222604 I

Email Zafar-Ahmad.Usmani@sa.gov.au
Background: Despite the high prevalence of anxiety in COPD patients and its impact on quality of life, evidence to support the effectiveness of various anxiety treatment options is insufficient, leading to the need for further research in this field.

Aim: The aim of this study was to assess the efficacy and safety of paroxetine for the management of anxiety in COPD and the impact of treatment on patients' quality of life and rate of hospitalization.

Patients and methods: In a double-blind, randomized, controlled trial, COPD patients were allocated into groups that either received paroxetine $20 \mathrm{mg}$ or placebo pills daily, for four months. Differences in outcomes were assessed based on an intention-to-treat analysis using linear mixed effects models. A chi-square test was used to compare the number of COPD-related admissions. Results: Thirty-eight participants were recruited. Twenty-two of these completed the trial. A clinically and statistically significant reduction was noted in anxiety symptoms after four months of treatment compared to the placebo. Clinically important improvement was noted in depression symptoms, with no statistically significant differences in walking distance or quality-of-life measure outcomes. The intervention group had less COPD-related admissions compared to the placebo group but experienced medication-related side effects.

Conclusion: Treatment with paroxetine significantly improved anxiety levels, but this difference did not translate into improved quality of life at four months follow-up.

Keywords: anxiety, COPD, pharmacotherapy, paroxetine, BAI, emphysema

\section{Introduction}

COPD is one of the most common chronic medical conditions worldwide with a prevalence range of $7.8-19.7 \%{ }^{1}$ Importantly, this estimate is predicted to double by $2050 .^{2}$ Anxiety and depression are common comorbidities of people with COPD, which are frequently undertreated or not treated at all. ${ }^{3}$ Anxiety is two- to threefold more common in COPD patients $\left(10-15.8 \%{ }^{4}\right)$ as compared to the general population, with a lifetime prevalence of $5 \% .{ }^{5}$

There are various physiological and pathological mechanisms that could be responsible for the increased levels of anxiety and panic in people with COPD. ${ }^{6,7}$ For example, COPD individuals frequently experience dyspnea and palpitations, which could be misinterpreted as serious or catastrophic events by the brain or body, leading to the creation of a positive feedback loop resulting in anxiety and panic., ${ }^{8,9}$ 
Coexisting anxiety among people with COPD is commonly associated with a deterioration in quality of life, ${ }^{10}$ self-management, ${ }^{11}$ exercise performance, ${ }^{12}$ and an increase in medical symptom reporting, ${ }^{13}$ illness exacerbations, ${ }^{12}$ hospitalizations, ${ }^{14}$ length of hospital stay, ${ }^{15}$ mortality, ${ }^{16}$ and health care costs. ${ }^{13}$ The impact of anxiety and depression on health-related outcomes in these individuals is independent of other confounding factors such as general health status, comorbidities, and disease severity. ${ }^{4}$ It has even been reported that the functional status of COPD patients is more dependent on their emotional status as compared to the actual severity of COPD itself. ${ }^{17,18}$

In clinical practice, pharmacological therapies are usually used as first-line therapy to treat anxiety in people with COPD. However, there is limited evidence to support this practice within the COPD population. ${ }^{19,20}$ There is currently a lack of randomized controlled trials to assess the effects of pharmacological interventions in people with COPD. The studies which are available have methodological limitations in terms of small sample size, large dropout rates, or a short follow-up period. ${ }^{19,21}$

Despite the lack of evidence, selective serotonin reuptake inhibitors (SSRIs) have been widely used in clinical practice for treating depression and anxiety in people with COPD. In our published Cochrane systematic review, we identified a potentially useful clinical improvement favoring the use of SSRIs over that of placebo for controlling anxiety in an underpowered study, while little or no difference was found for the other classes of medications. ${ }^{21}$ Unlike other SSRIs, paroxetine is indicated for the treatment of both depression and anxiety, ${ }^{22,23}$ which is of relevance in COPD sufferers. Another study of paroxetine in people with COPD and comorbid depression showed significant improvements in depressive symptoms, exercise capacity, and quality of life at three months follow-up. ${ }^{24}$ However, this study was underpowered, and the authors concluded that a larger, double-blind trial with a relatively longer treatment period was indicated. As such, a case can be made that a study of robust design and appropriate power is warranted.

Our study tested the hypothesis that people with COPD and clinically significant anxiety, recruited from public hospitals, who are prescribed $20 \mathrm{mg}$ of paroxetine (Pharmaceutical Packaging Professionals Pty Ltd., Port Melbourne, VIC, Australia) daily for 16 weeks will:

(Hypothesis 1: principle hypothesis) have a significant reduction in their anxiety symptoms measured by Beck Anxiety Inventory (BAI) as compared with the placebo at four months follow-up, and
(Hypothesis 2) will experience improved quality of life measured by Chronic Respiratory Questionnaire (CRQ), and exercise capacity measured by six-minute walk distance (6MWD), and

(Hypothesis 3) have reduced hospital visits.

\section{Methods}

\section{Ethical approval}

The trial was approved by the Human Research and Ethics Committee of The Queen Elizabeth Hospital, Adelaide, SA (HREC Ref \# 2012012). The trial was registered with the Australian and New Zealand Clinical Trials Registry (Trial ID: ACTRN12613000458730) in April 2013, and participant recruitment began in June 2013.

\section{Design}

The trial was double blind, placebo controlled, with randomization to either:

1. paroxetine $20 \mathrm{mg}$ daily (four-month course);

2. placebo tablets of identical appearance (four-month course).

\section{Hospital sampling frame and recruitment} The sampling frame included four Adelaide Hospitals, The Queen Elizabeth Hospital (TQEH), Repatriation General Hospital (RGH), Flinders Medical Centre (FMC), and the Royal Adelaide Hospital (RAH), which collectively have more than 40,000 admissions per year. Of these, at least 4,000 admissions are for patients diagnosed with COPD.

\section{Inclusion criteria}

Patients who met the following criteria were included in the trial: adults over 40 years of age, diagnosed with any class of COPD of any severity (by a medical consultant or by Global initiative for chronic Obstructive Lung Disease [GOLD] criteria: post-bronchodilator forced expiratory volume in one second $[\mathrm{FEV} 1]$ /forced vital capacity $<0.70^{25}$ ), and recognized as having clinically significant anxiety, with a score of $>15$ as assessed using BAI. ${ }^{26}$

The BAI has been validated for use with older medical patients, ${ }^{27}$ and the scale possesses strong psychometric properties related to internal consistency, validity, and test-retest reliability. ${ }^{28}$ The BAI can be used to assess and establish a baseline anxiety level, as a diagnostic aid, and also as a posttreatment outcome measure.

The BAI is a 21-question multiple-choice self-report inventory used for measuring the severity of anxiety. Total 
scores ranging from 0 to 7 are indicative of minimal anxiety, 8-15 of mild anxiety, 16-25 of moderate anxiety, and 26-63 of severe anxiety. However, it is worth noting that the BAI includes numerous somatic symptoms that may represent COPD rather than anxiety, so COPD patients with anxiety scores at the very mild end of the scale should be treated cautiously. A score of $>15$ was categorized as moderate or severe anxiety ${ }^{28}$ and has been used in previous studies as the "cutoff" for anxiety in people with COPD ${ }^{17}$ hence, a BAI score of $>15$ was chosen to define clinical anxiety.

\section{Exclusion criteria}

Patients with any of the following criteria were excluded: current or recent (within a week) exacerbation of COPD; severe dementia or significant cognitive impairment, terminal cancer, or other concurrent significant psychological disease (e.g., schizophrenia or suicidal ideation); history of current or recent (within last two weeks) use of monoamine oxidase inhibitors; Prolonged QT interval $(>440 \mathrm{~ms}$ in males and $>460 \mathrm{~ms}$ in females) on ECG; current pregnancy or lactation; severe liver, kidney, cardiovascular, or locomotor disease; uncontrolled epilepsy or previous history of SSRI intolerance.

\section{Randomization, allocation concealment, and blinding}

All the participants were provided with written informed consent and signed the consent prior to being enrolled in the study. Randomization was accomplished by a random number generator computer software program, by an external researcher unrelated to the trial. Allocation concealment and blinding were maintained for patients, investigators, research/ data collection staff, and outcome assessors by using an external pharmacy dispensing company to package the study medication as per the randomization schedule into identical containers, which were consecutively numbered. This medication was then dispensed by the hospital pharmacy as each new participant was recruited. However, if a subject experienced a serious adverse event, the treatment was ceased, and the code was broken by a research pharmacist unrelated to the trial, resulting in all trial staff remaining blinded.

\section{Intervention delivery and monitoring}

Research staff blinded to the treatment allocation commenced patients on $20 \mathrm{mg}$ of paroxetine or an identical placebo pill during their first visit for baseline measurements. Patients were provided with contact details of research personnel for notification of adverse events. The medication was provided for 16 weeks with a weekly phone call for the first four weeks to identify and address any side effects or adverse events. This procedure is summarized in Figure 1.

\section{Data collection}

\section{Baseline questionnaires}

Patient demographic characteristics including age, gender, ethnicity, and marital and smoking status were collected. Details regarding the diagnosis and severity of COPD and other medical comorbidities were also documented, and a detailed note was made of all concomitant medications. Primary outcome of anxiety was measured using BAI. Secondary outcomes included depression measured using Beck Depression Inventory (BDI), ${ }^{29}$ quality of life assessed via $\mathrm{CRQ},{ }^{30}$ and dyspnea assessed using modified Medical Research Council dyspnea scale. ${ }^{31,32}$ Smoking status, 6MWD, and spirometry were also documented.

\section{Follow-up questionnaires}

Weekly phone calls were made for the first four weeks to assess compliance and any intervening management, and for adverse events monitoring. The prespecified outcomes of anxiety, depression, quality of life, dyspnea score, 6MWD, spirometry, and hospital utilization were reassessed at four months during a face-to-face visit.

\section{Withdrawal criteria}

The withdrawal criteria included any clinically significant adverse event that was considered related to the study and patients' unwillingness to continue further with the trial.

\section{Statistical analysis}

Very few previous studies were available to guide the original power calculation. One study had shown an effect size of $1.0,3^{33}$ however, we chose a more conservative effect size estimate of 0.5 , based on a linear mixed effects model with two time points, a power of $80 \%$, a type 1 error of 0.05 , and an expected correlation between baseline and four-month results of 0.4 . This required a sample size of 50 patients in each study arm. However, after three years of an intense recruitment campaign, only 38 participants could be recruited and were randomized to either placebo or intervention group. An intention-to-treat analysis was conducted by the trial statistician to determine if significance was reached and the study could be discontinued. Linear mixed effects models were used to assess change score by group. The fixed factors consisted of group, time, and a group-time interaction term, 


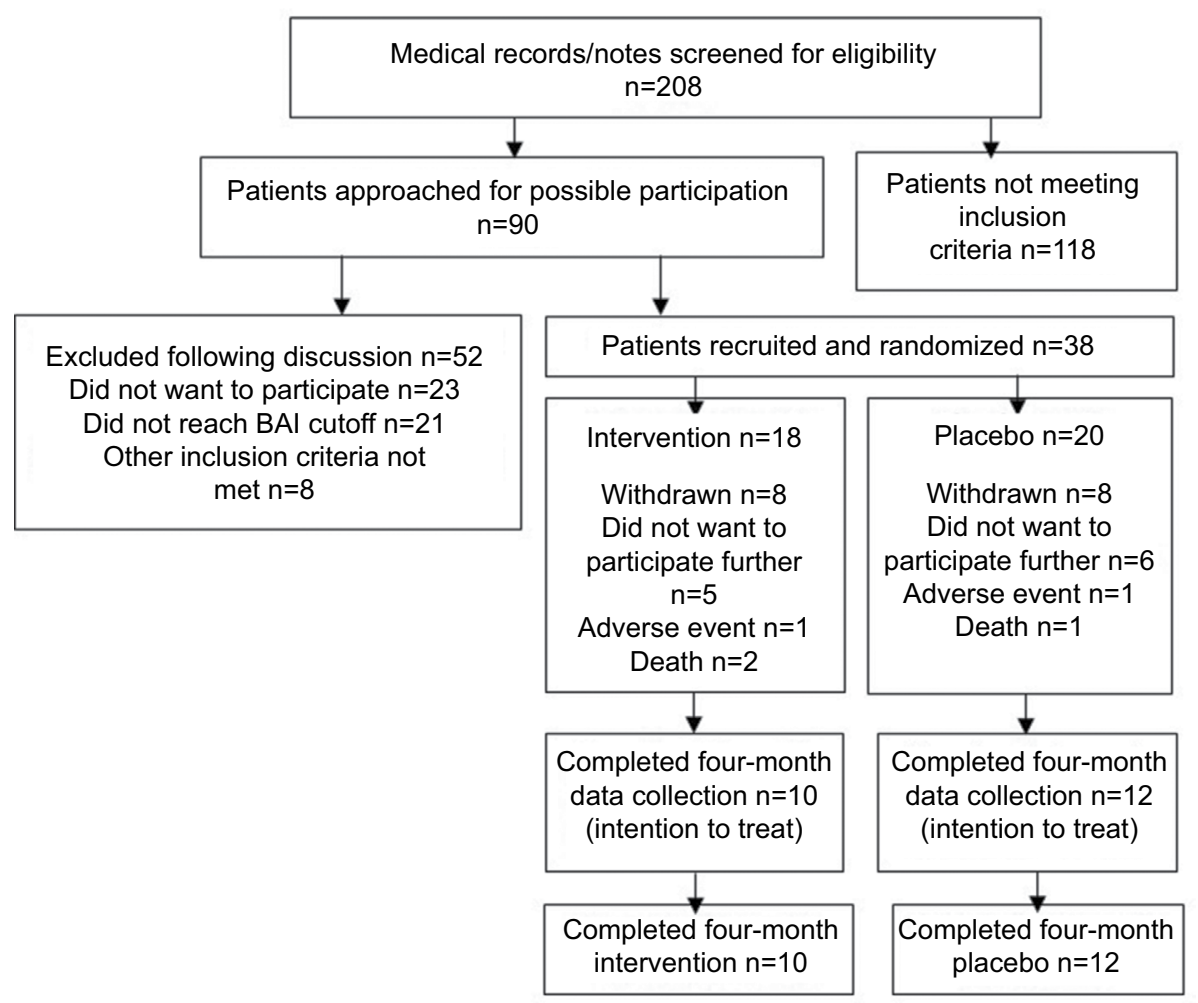

Figure I Flowchart of the study.

Abbreviation: BAI, Beck Anxiety Inventory.

the latter being the basis for a formal test of the intervention. A chi-square test was used to compare the number of COPDrelated admissions by group.

\section{Results}

\section{Participants}

A total of 208 patients were screened for eligibility (Figure 1). More than half of these did not meet the prespecified inclusion criteria. Ninety patients were approached for enrollment, and more than half of these could not be included primarily due to a lack of interest for participation or because their BAI score was $<15$. Thirty-eight participants were enrolled during three years of recruitment.

Demographics for this population were similar between groups with no statistically or clinically significant differences observed (Table 1). Two participants in each group had past history of depression. The mean FEV1 in the intervention group was $44.7 \%$ versus mean FEV1 in placebo group of $35.8 \%$, however, from a clinical perspective, both averages were within the range for severe COPD or GOLD stage III classification.

Eighteen participants were randomized to the intervention group, with eight of these lost to follow-up and therefore not available for the four-month questionnaire. Out of these
Table I Baseline demographic characteristics

\begin{tabular}{|c|c|c|}
\hline Demographics & $\begin{array}{l}\text { Intervention } \\
(n=18)\end{array}$ & $\begin{array}{l}\text { Placebo } \\
(n=20)\end{array}$ \\
\hline Age (years) & $70.6(9.0)$ & $67.6(7.7)$ \\
\hline Males:females (n) & $9: 9$ & $11: 9$ \\
\hline COPD severity (FEVI\%) & $44.7(18.0)$ & $35.8(16.1)$ \\
\hline BMI $\left(\mathrm{kg} / \mathrm{m}^{2}\right)$ & $25.7(6.6)$ & $28.5(5.5)$ \\
\hline Pulmonary rehabilitation: yes (n, \%) & $2(11.1 \%)$ & $3(15.0 \%)$ \\
\hline \multicolumn{3}{|l|}{ Ethnicity (n, \%) } \\
\hline Caucasian & $18(100 \%)$ & $20(100 \%)$ \\
\hline Other & 0 & 0 \\
\hline \multicolumn{3}{|l|}{ Educational attainment (n, \%) } \\
\hline High school or less & 17 (94.4\%) & $18(90 \%)$ \\
\hline Some college & 0 & 0 \\
\hline College degree or higher & I (5.6\%) & $2(10.0 \%)$ \\
\hline \multicolumn{3}{|l|}{ Household income (n, \%) } \\
\hline Low income ( $\leq \$ 20$ kp.a.) & $10(55.6 \%)$ & $12(60.0 \%)$ \\
\hline Medium income $(\$ 20-\$ 50$ kp.a. $)$ & $4(22.2 \%)$ & $5(25.0 \%)$ \\
\hline Unknown income & $4(22.2 \%)$ & $3(15.0 \%)$ \\
\hline \multicolumn{3}{|l|}{ Smoking status (n, \%) } \\
\hline Current smoker & $6(33.3 \%)$ & $5(33.3 \%)$ \\
\hline Ex-smoker & $18(66.7 \%)$ & $15(66.7 \%)$ \\
\hline
\end{tabular}

Note: Data are presented as mean (standard deviation) unless otherwise specified. Abbreviations: FEVI, forced expiratory volume in one second; BMI, body mass index; kp.a., thousand per annum.

eight patients, one suffered severe nausea and dizziness as a side effect of the intervention, two passed away for reasons unrelated to the trial, and five refused follow-ups for various 
reasons (two changed their mind and did not wish to continue, one developed other health priorities, one reported significant worsening of anxiety, and one experienced significant deterioration of health).

Twenty participants were randomized to the control group. Eight of these were lost to follow-up and were not available for the four-month questionnaire. Of these eight patients, one suffered severe headaches as a side effect of placebo, one passed away for reasons unrelated to the trial, and six refused follow-ups for various reasons (two changed their minds and did not wish to continue, two had general deterioration of health, one developed other health priorities, and one reported significant worsening of anxiety and did not wish to continue). No significant difference was noted in terms of COPD severity between the patients who completed the trial versus those who lost the follow-up in either of the groups.

Subsequently, data from 10 participants in the intervention and 12 participants in the placebo group were available for the intention-to-treat analysis. Of these subjects, seven in the intervention and six in the placebo group completed the entire course of 120 capsules. The number of capsules returned by the other participants was minimal and ranged between 4 and 17. However, it is expected that unintentional missed doses would not cause any significant clinical impact, particularly in the setting of proper randomization and blinding.

\section{Treatment effect}

For the primary outcome of anxiety (BAI), participants in the intervention group had a greater improvement after four months compared to the placebo group (Table 2). The difference was both statistically and clinically significant $(p=0.007)$. Mean change (reduction) of 11.9 units of BAI was noted in the intervention group versus 3.16 units in the placebo group.

Clinically important improvement in the treatment group (with a mean change of 4.5 units of BDI) was noted for depression symptoms compared to the placebo group. Neither clinical nor statistical difference was noted for dyspnea, walking distance, or quality-of-life outcomes.

There were fewer COPD-related hospital admissions in the intervention group compared to the placebo group during the four-month treatment phase ( $1 / 10$ and $6 / 12$, respectively; $p=0.050$ ). However, this was based on a small number of admissions and shorter follow-up period. More side effects were reported in the intervention arm compared to the placebo arm (Table 3).

\section{Discussion}

COPD is one of the most common medical conditions worldwide, and comorbid anxiety is frequently seen in people suffering from COPD. Anxiety when associated with COPD also has a significant impact on patients' exercise capacity and quality of life. There is evidence that the rate of

Table 3 COPD-related hospital admissions and adverse events during treatment period

\begin{tabular}{lll}
\hline Side effects & Intervention & Placebo \\
Headache & $\mathrm{I}$ & $\mathrm{I}$ \\
Heart burn & $\mathrm{I}$ & 0 \\
Insomnia & $\mathrm{I}$ & 0 \\
Itch & 0 & $\mathrm{I}$ \\
Nausea & $\mathrm{I}$ & 0 \\
Stomach upset & $\mathrm{I}$ & 0 \\
Shakes & $\mathrm{I}$ & 0 \\
Tiredness & 0 & $\mathrm{I}$ \\
Vertigo & 0 & $\mathrm{I}$ \\
Total adverse events & 6 & 4 \\
\hline
\end{tabular}

Table 2 Outcome scores at baseline and follow-up with differences between groups

\begin{tabular}{|c|c|c|c|c|c|c|c|}
\hline \multirow[t]{2}{*}{ Outcome measured } & \multicolumn{3}{|c|}{ Intervention $(n=10)$} & \multicolumn{3}{|c|}{ Placebo $(n=12)$} & \multirow[t]{2}{*}{ p-value* } \\
\hline & Baseline & Follow-up & Change & Baseline & Follow-up & Change & \\
\hline Anxiety (BAI) & $26.80(7.4)$ & 14.90 (8.8) & -11.9 & $25.25(8.2)$ & $22.09(11.8)$ & -3.16 & 0.007 \\
\hline Depression (BDI) & $16.20(10.7)$ & $\mathrm{II} .70(10.5)$ & -4.5 & $14.58(8.2)$ & $15.92(10.6)$ & 1.34 & 0.144 \\
\hline Dyspnea (mMRC dyspnea scale) & $2.00(1.2)$ & $2.00(1.2)$ & 0.0 & $2.17(1.1)$ & $2.42(0.9)$ & 0.25 & 0.684 \\
\hline 6MWD (m) & $311.90(132.9)$ & $329.13(115.7)$ & 17.23 & $294.45(115.7)$ & $354.56(62.0)$ & 60.11 & 0.301 \\
\hline \multicolumn{8}{|l|}{ CRQ } \\
\hline Dyspnea & $3.62(0.9)$ & $3.80(1.4)$ & 0.18 & $3.50(1.2)$ & $3.48(1.4)$ & -0.02 & 0.775 \\
\hline Fatigue & $3.42(1.0)$ & $3.83(1.3)$ & 0.41 & $3.42(1.4)$ & $2.84(0.7)$ & -0.58 & 0.089 \\
\hline Emotional & $3.68(1.2)$ & $4.83(1.1)$ & -1.55 & $3.76(1.4)$ & $4.19(1.3)$ & 0.43 & 0.196 \\
\hline Mastery & $3.25(1.3)$ & $4.25(1.3)$ & 1.0 & $3.52(1.7)$ & $4.00(0.8)$ & 0.48 & 0.270 \\
\hline
\end{tabular}

Notes: Data are presented as mean (standard deviation). *Based on the statistical significance of the group-time interaction term in the linear mixed effects model. Abbreviations: BAI, Beck Anxiety Inventory; BDI, Beck Depression Inventory; m, meters; mMRC, modified Medical Research Council; 6MWD, six-minute walk distance; CRQ, Chronic Respiratory Questionnaire. 
hospitalization is also increased among these patients, possibly due to noninfective presentations or primary presentations because of panic and then being found to have low oxygen saturation due to underlying COPD and admitted to hospital.

Despite the impact of psychological comorbidities, the major focus of clinical practice and research still focuses on improvement in lung function, rather than patients' emotional and psychological well-being.

As discussed above, previous trials for the pharmacological treatment of anxiety in people with COPD were of small sample size; hence, we decided to conduct a larger clinical trial. However, the difficulty we faced in terms of recruitment and retention of the participants has further confirmed the complex nature and management issue surrounding these patients and may explain the small sample size of previous trials.

This study has shown that a four-month course of paroxetine reduces anxiety levels in people with COPD compared to placebo, albeit based on a small sample size. It is important to note that anxiety and depression often coexist, and although not statistically significant, our study demonstrated a clinically significant improvement in depression scores for the treatment arm, measured by BDI. However, at four months, these improvements in anxiety and depression scores did not translate into improvement in exercise capacity or quality of life; a longer duration of treatment may be required to prompt this. Another possible explanation for this lack of effect on exercise capacity and quality of life could be that though the medication has calmed the patients, it has not modified their thought process and behavior, an effect which could be achieved by adding a psychological intervention. Both the study arms experienced similar rates of withdrawal; however, side effects of nausea, stomach upset, and shakes were more common in the treatment group; these are common and expected side effects of SSRIs. The incidence of hospital admission was less in the treatment group compared to placebo, though the follow-up period was short. Hence, we remain cautious about drawing firm conclusions on this. One explanation for the reduced admission rate could be associated with a reduction in anxiety levels resulting in a lesser number of self-presentations due to stress and panic.

We observed a significant reduction in anxiety levels and some reduction in depression and hospitalization rates over a short follow-up period. It is possible that the large change in BAI may be related to the difference in the underlying nature of the anxiety disorder; for example, the intervention arm may have had a larger proportion of participants with panic disorder. However, we did not perform any stratification during the randomization of participants based on their levels of anxiety or the nature of their underlying anxiety disorder. We believe one of the most important messages of this study comes from issues with patient recruitment and retention, being that this particular group of people with COPD are difficult to engage due to their underlying panic and stress. This is also likely to be the underlying reason for these individuals demonstrating a low threshold for discontinuing their medication and becoming lost from close clinical follow-up. We recommend that further research into the problem of anxiety in people with COPD be focused on psychological interventions, for example, cognitive behavioral therapy, as this type of intervention could modify patients' behavior and thought process, without the introduction of side effects and hence may be a better long-term management option for this population.

\section{Acknowledgments}

The authors wish to acknowledge Harshani Jayasinghe and Malcolm Brinn for their assistance with patient recruitment and data collection and Zoe Kopsaftis for assistance with manuscript editing. They also wish to thank June Challen from the Clinical Trials Unit at TQEH pharmacy for assistance with blinding and randomization along with Pamela Kidd and Donna Keatley from the Pulmonary Function Laboratory at TQEH Respiratory Medicine for assistance with performing various tests. They wish to thank Dr Paul Cafarella (RGH), Dr Jeff Bowden (FMC), Dr Ral Antic (RAH), Prof Mark Holmes (RAH), Mrs Karen Royals (TQEH), and Mrs Mary Young (RAH) for their assistance with recruitment at various sites. This study was funded by the Department of Respiratory Medicine, The Queen Elizabeth Hospital, Adelaide, SA, Australia.

\section{Disclosure}

The authors report no conflicts of interest in this work.

\section{References}

1. Global Initiative for Chronic Obstructive Lung Disease (GOLD). Global strategy for the diagnosis, management and prevention of COPD. 2016. Available from: http://goldcopd.org/. Accessed 2018.

2. Access Economics Pty Limited. Economic impact of COPD and cost effective solutions. The Australian Lung Foundation; 2008.

3. Cully JA, Graham DP, Stanley MA, et al. Quality of life in patients with chronic obstructive pulmonary disease and comorbid anxiety or depression. Psychosomatics. 2006;47(4):312-319.

4. Brenes GA. Anxiety and chronic obstructive pulmonary disease: prevalence, impact and treatment. Psychosom Med. 2003;65(6):963-970.

5. Wittchen HU, Hoyer J. Generalised anxiety disorder: nature and course. J Clin Psychiatry. 2001;62 Suppl 11:15-19.

6. Ley R. Agoraphobia, the panic attack and the hyperventilation syndrome. Behav Res Ther. 1985;23(1):79-81. 
7. Perna G, Caldirola D, Bellodi L. Panic disorder: from respiration to the homeostatic brain. Acta Neuropsychiatrica. 2004;16(2):57-67.

8. Clark D. A cognitive approach to panic. Behav Res Ther. 1986;24(4): 461-470.

9. Livermore N, Sharpe L, McKenzie D. Panic attacks and panic disorder in chronic obstructive pulmonary disease: a cognitive behavioral perspective. Respir Med. 2010;104(9):1246-1253.

10. Giardino ND, Curtis JL, Andrei AC, et al; NETT research group. Anxiety is associated with diminished exercise performance and quality of life in severe emphysema: a cross-sectional study. Respir Res. 2010; 11:29.

11. Dowson CA, Town GI, Frampton C, Mulder RT. Psychopathology and illness beliefs influence COPD self-management. Psychosom Res. 2004;56(3):333-340.

12. Eisner MD, Blanc PD, Yelin EH, et al. Influence of anxiety on health outcomes in COPD. Thorax. 2010;65(3):229-234.

13. Katon W, Lin EH, Kroenke K. The association of depression and anxiety with medical symptom burden in patients with chronic medical illness. Gen Hosp Psychiatry. 2007;29(2):147-155.

14. Gudmundsson G, Gislason T, Janson C, et al. Risk factors for rehospitalisation in COPD: role of health status, anxiety and depression. Eur Res J. 2005;26(3):414-419.

15. Xu W, Collet JP, Shapiro S, et al. Independent effect of depression and anxiety on chronic obstructive pulmonary disease exacerbations and hospitalizations. Am J Respir Crit Care Med. 2008;178(9): 913-920.

16. Celli BR, Cote CG, Lareay SC, Meek PM. Predictors of survival in COPD: more than just the FEV1. Respir Med. 2008;102 Suppl 1: S27-S35.

17. Kim HF, Kunik ME, Molinari VA, et al. Functional impairment in COPD patients: the impact of anxiety and depression. Psychosomatics. 2000;41(6):465-471

18. Atlantis EF, Fahey P, Cochrane B, Smith S. Bidirectional associations between clinically relevant depression or anxiety and COPD: a systematic review and meta-analysis. Chest. 2013;144(3):766-777.

19. Cafarella PA, Effing TW, Usmani ZA, Frith PA. Treatments for anxiety and depression in patients with COPD: a literature review. Respirology. 2012;17(4):627-638.

20. Usmani ZA, Carson KV, Heslop K, Esterman AJ, De Soyza A, Smith BJ. Psychological therapies for the treatment of anxiety disorders in chronic obstructive pulmonary disease. Cochrane Database Syst Rev. 2017;3:CD010673.
21. Usmani ZA, Carson KV, Cheng JN, Esterman AJ, Smith BJ. Pharmacological interventions for the treatment of anxiety disorders in chronic obstructive pulmonary disease. Cochrane Database Syst Rev. 2011;11:CD008483.

22. Baldwin DS, Anderson IM, Nutt DJ, et al; British Association for Psychopharmacology. Evidence-based guidelines for the pharmacological treatment of anxiety disorders: recommendations from the British Association for Psychopharmacology. J Psychopharmacol. 2005;19(6):567-596.

23. MIMS USA. Paroxetine. UBM Medica; 2011.

24. Eiser N, Harte R, Spiros K, Phillips C, Isaac MT. Effect of treating depression on quality-of-life and exercise tolerance in severe COPD. COPD. 2005;2(2):233-241.

25. Global Initiative for Chronic Obstructive Lung Disease. Global strategy for the diagnosis, management, and prevention of chronic obstructive pulmonary disease: executive summary. 2006. Available from: goldcopd.org/wp-content/uploads/2018/.../WMS-GOLD-2018-FebFinal-to-print-v2.pdf.

26. Steer RA, Ranieri WF, Beck AT, Clark DA. Further evidence for the validity of the Beck Anxiety Inventory with psychiatric outpatients. J Anxiety Disord. 1993;7(3):195-205.

27. Wetherell JL, Arean PA. Psychometric evaluation of the Beck Anxiety Inventory with older medical patients. Psychol Assess. 1997;9(2):136-144

28. Beck AT, Epstein N, Brown G, Steer RA. An inventory for measuring clinical anxiety: psychometric properties. J Consult Clin Psychol. 1988;56(6):893-897.

29. Beck AT, Ward CH, Mendelson M, Mock J, Erbaugh J. An inventory for measuring depression. Arch Gen Psychiatry. 1961;4:561-571.

30. Guyatt GH, Berman LB, Townsend M, Pugsley SO, Chambers LW. A measure of quality of life for clinical trials in chronic lung disease. Thorax. 1987;42(10):773-780.

31. Hajiro T, Nishimura K, Tsukino M, Ikeda A, Koyama H, Izumi T. Analysis of clinical methods used to evaluate dyspnea in patients with chronic obstructive pulmonary disease. Am J Respir Crit Care Med. 1998;158(4):1185-1190.

32. Mahler DA, Wells CK. Evaluation of clinical methods for rating dyspnea. Chest. 1988;93(3):580-586.

33. Hynninen MJ, Bjerke N, Pallesen S, Bakke PS, Nordhus IH. A randomized controlled trial of cognitive behavioral therapy for anxiety and depression in COPD. Respir Med. 2010;104(7):986-994.
Journal of Multidisciplinary Healthcare

\section{Publish your work in this journal}

The Journal of Multidisciplinary Healthcare is an international, peerreviewed open-access journal that aims to represent and publish research in healthcare areas delivered by practitioners of different disciplines. This includes studies and reviews conducted by multidisciplinary teams as well as research which evaluates the results or conduct of such teams or health
Dovepress

care processes in general. The journal covers a very wide range of areas and welcomes submissions from practitioners at all levels, from all over the world The manuscript management system is completely online and includes a very quick and fair peer-review system. Visit http://www.dovepress.com/ testimonials.php to read real quotes from published authors. 